\title{
Gravity with dynamical torsion
}

\author{
M. O. Katanaev* \\ Steklov Mathematical Institute of Russian Academy of Sciences, \\ 8 Gubkina St., Moscow 119991, Russia
}

September 21, 2021

\begin{abstract}
We propose four simple Lagrangians for gravity models with dynamical torsion which are free from ghosts and tachyons. The torsion propagates two massive or massless particles of spin $1^{ \pm}$and $0^{ \pm}$besides the massless graviton $2^{+}$propagated by metric.
\end{abstract}

\section{Introduction}

Introduction of dynamical torsion in general relativity is the natural geometric generalization of Einstein's gravity. It is the subject of research for many years. Physical motivations and earlier references can be found in [1, 2, 3, 4, 5]. In spite of much efforts, we still do not have a generally acknowledged action for such model. It is usually assumed that gravity interactions are described by metric propagating spin $2^{+}$massless gravitons, whereas torsion describes massive or massless particles of spin $2^{ \pm}, 1^{ \pm}$, or $0^{ \pm}$. To answer the crucial question whether torsion can yield a physically acceptable generalization, we need a model but it is missing, rather we have too many proposals and cannot make a unique choice. To my mind, the main problem here is the mathematical complexity.

Torsion has three tensor indices and can propagate several massive or massless particles of spin not higher then 2. From physical standpoint, the model must not include ghosts and tachyons. A number of such models were proposed forty years ago [6, 7, 8, 9], but they are not analyzed until now if full detail, mainly, because of technical difficulties. Moreover, these models were found within a certain class of Lagrangians which do not exhaust all possibilities. In the present paper, we consider another class of dynamical torsion models without higher derivatives, considering metric and torsion as independent variables, and found four Lagrangians without ghosts and tachyons. The final answer seems to be much simpler and transparent then previous proposals.

\section{General Lagrangian}

We consider a space-time manifold $\mathbb{M}$ with local coordinates $x^{\alpha}, \alpha=0,1,2,3$, and the Riemann-Cartan geometry. The geometry can be described either by metric $g_{\alpha \beta}$ and

*E-mail: katanaev@mi-ras.ru 
torsion $T_{\alpha \beta}{ }^{\gamma}=-T_{\beta \alpha}{ }^{\gamma}$ or, equivalently, by Cartan variables: vierbein $e_{\alpha}{ }^{a}$ and Lorentz connection $\omega_{\alpha}^{a b}=-\omega_{\alpha}{ }^{b a}, a, b=0,1,2,3$.

As usual, a vierbein defines unique metric

$$
g_{\alpha \beta}:=e_{\alpha}^{a} e_{\beta}^{b} \eta_{a b}, \quad \eta_{a b}:=\operatorname{diag}(+---),
$$

and the Lorentz connection is defined through the affine connection $\Gamma_{\alpha \beta}{ }^{\gamma}$ by the vielbein postulate

$$
\nabla_{\alpha} e_{\beta}^{a}=\partial_{\alpha} e_{\beta}^{a}-\Gamma_{\alpha \beta}^{\gamma} e_{\gamma}^{a}+e_{\beta}^{b} \omega_{\alpha b}^{a}=0 .
$$

If the vierbein is given, then this equation provides the one-to-one correspondence between Lorentz connection and the torsion $T_{\alpha \beta}{ }^{\gamma}:=\Gamma_{\alpha \beta}{ }^{\gamma}-\Gamma_{\beta \alpha}{ }^{\gamma}$.

The vierbein has 6 components more then the metric due to the extra symmetry with respect to local Lorentz transformations acting on Latin indices $a, b, \ldots$ In what follows, transformation of Latin indices into Greek ones and vice versa is always performed by the vierbein and its inverse $e^{\alpha}{ }_{a}$. Moreover we assume that coordinates are chosen in such a way that the coordinate $x^{0}$ is timelike and all sections $x^{0}=$ const are spacelike.

Torsion and curvature have the following expressions in terms of Cartan variables

$$
\begin{aligned}
T_{\alpha \beta}{ }^{a} & =\partial_{\alpha} e_{\beta}{ }^{a}-e_{\alpha}{ }^{b} \omega_{\beta b}{ }^{a}-(\alpha \leftrightarrow \beta), \\
R_{\alpha \beta a}{ }^{b} & =\partial_{\alpha} \omega_{\beta a}{ }^{b}-\omega_{\alpha a}{ }^{c} \omega_{\beta c}{ }^{b}-(\alpha \leftrightarrow \beta) .
\end{aligned}
$$

If we want vierbein and Lorentz connection to be really independent propagating fields then they must satisfy differential equations of motion. Let us require the field equations to be covariant and of second order. Then the Lagrangian should be quadratic in curvature and torsion. The general form of such Lagrangian is

$$
\begin{aligned}
\frac{1}{\sqrt{|g|} L=} & \kappa R-\frac{1}{4} T^{a b c}\left(\beta_{1} T_{a b c}+\beta_{2} T_{c a b}+\beta_{3} \eta_{a c} T_{b}\right)- \\
& -\frac{1}{4} R^{a b c d}\left(\gamma_{1} R_{a b c d}+\gamma_{2} R_{c d a b}+\gamma_{3} R_{a c b d}+\gamma_{4} \eta_{b d} R_{a c}+\gamma_{5} \eta_{b d} R_{c a}\right)-2 \Lambda,
\end{aligned}
$$

where $T_{b}:=T_{a b}{ }^{b}$ is the trace of torsion, $R_{a b}:=R_{a c b}{ }^{c}$ and $R:=R_{a}{ }^{a}$ are the Ricci tensor and scalar curvature, respectively; $\kappa, \beta_{1,2,3}$, and $\gamma_{1,2,3,4,5}$ are coupling constants, and $\Lambda$ is a cosmological constant. We do not have to add the scalar curvature $\widetilde{R}(e)$ built from Christoffel's symbols for zero torsion due to the identity

$$
R+\frac{1}{4} T_{\alpha \beta \gamma} T^{\alpha \beta \gamma}-\frac{1}{2} T_{\alpha \beta \gamma} T^{\gamma \alpha \beta}-T_{\alpha} T^{\alpha}-\frac{2}{\sqrt{|g|}} \partial_{\alpha}\left(\sqrt{|g|} T^{\alpha}\right)=\widetilde{R} .
$$

Here and in what follows the tilde sign marks geometric notions constructed entirely from metric for zero torsion.

There are three independent invariants quadratic in torsion in Lagrangian (4) because torsion has three irreducible components with respect to the Lorentz group. Curvature tensor in Riemann-Cartan geometry has six irreducible components but we added only five in Eq.(4) due to the Gauss-Bonnet type identity

$$
R_{a b c d} R^{c d a b}-4 R_{a b} R^{b a}+R^{2} \equiv \widetilde{\nabla}_{\alpha} X^{\alpha},
$$

where $X^{\alpha}$ are components of some vector. 
Several models without ghosts and tachyons were found within the class of Lagrangians (41) in [7, 8, 9], and the analysis is rather complicated. There is the additional problem. Lagrangian (4) is not the most general one in dynamical torsion models, because the existence or absence of higher derivatives depend on the chosen independent variables. For example, transformation of the Lorentz connection into torsion tensor by Eq.(1) involves derivatives of the vierbein. Therefore Lagrangian (44) is a higher derivative one for the vierbein-torsion variables.

In what follows, we need the decomposition of torsion tensor $T_{a b c}$ into irreducible pieces with respect to the Lorentz group. To this end we extract the trace $T_{a}$ and totally antisymmetric part $T_{[a b c]}$ :

$$
T_{a b c}=S_{a b c}+\frac{1}{3}\left(\eta_{a c} T_{b}-\eta_{b c} T_{a}\right)+T_{[a b c]},
$$

where

$$
T_{b}:=T_{a b}^{a}, \quad T_{[a b c]}:=\frac{1}{3}\left(T_{a b c}+T_{c a b}+T_{b c a}\right) .
$$

The irreducible component $S_{a b c}$ is defined by the algebraic relations:

$$
S_{a b c}=-S_{b a c}, \quad S_{a b}^{a}=0, \quad S_{a b c}+S_{b c a}+S_{c a b}=0 .
$$

In four dimensions, the totally antisymmetric component of torsion is parameterized by the pseudovector field $T^{* d}:=-\frac{1}{6} T_{a b c} \varepsilon^{a b c d}$, where $\varepsilon^{a b c d}$ is the totally antisymmetric tensor, $\varepsilon_{0123}=1$. Thus the torsion tensor has three irreducible components.

We change variables: $e_{\alpha}{ }^{a}, \omega_{\alpha}{ }^{a b} \mapsto e_{\alpha}{ }^{a}, T_{\alpha \beta \gamma}$. Now the quadratic torsion terms become simply the mass terms for torsion which can be easily analyzed. The transformation of variables restricts the possible choice of kinetic terms for the vierbein, if we do not want it to be mixed with the torsion. The corresponding invariant must have dimension $l^{-2}$, where $l$ is the dimension of length. We have only five tensors of this dimensionality in our disposal

$$
\widetilde{R}_{\alpha \beta \gamma \delta}, \quad R_{\alpha \beta \gamma \delta}, \quad \widetilde{\nabla}_{\alpha} T_{\beta \gamma \delta}, \quad \nabla_{\alpha} T_{\beta \gamma \delta}, \quad T_{\alpha \beta \gamma} T_{\delta \epsilon \zeta} .
$$

The invariants are built by different contractions of these tensors with the metric. We see that the only possible invariants are $\widetilde{R}$ and $\widetilde{\nabla}_{\alpha} T^{\alpha}$. The last term mixes kinetic term of the vierbein with torsion components. To simplify matters we postulate that this term must be absent in dynamical torsion theory. Thus we are left with the unique possibility: the Hilbert-Einstein action for the vierbein. Therefore, we further transform variables: $e_{\alpha}{ }^{a}, T_{\alpha \beta \gamma} \mapsto g_{\alpha \beta}, T_{\alpha \beta \gamma}$.

It is well known that metric in the Hilbert-Einstein action describes massless gravitons $2^{+}$, and the full symmetry under general coordinate transformations must be used for elimination of the unphysical degrees of freedom of the metric. Therefore the problem is reduced to choosing the Lagrangian for the torsion tensor, and, what is important, we cannot use general coordinate transformations to eliminate its degrees of freedom.

\section{3 (1+3)-decomposition of torsion}

Torsion tensor has three irreducible components with respect to the Lorentz group: $S_{a b c}$, $T_{a}$, and $T_{a}^{*}$. To analyse positive definiteness of quadratic forms of velocities and masses, we further decompose torsion components with respect to the rotational subgroup $\mathbb{S O}(3) \subset$ 
$\mathbb{S O}(1,3)$, because invariant quadratic forms for the Lorentz group are not positive or negative definite.

Positive definiteness of the quadratic form of torsion momenta or, equivalently, velocities depends only on the metric signature. Hence, we put $g_{\alpha \beta} \mapsto \eta_{a b}:=\operatorname{diag}(+---)$, i.e. we shall work in Minkowskian space. We perform the $(3+1)$-decomposition of all components: $(a)=(0, i), i:=1,2,3$. Space indices are denoted by Latin letters from the middle of the alphabet $i, j, \ldots:=1,2,3$, and summation over them is performed by the Euclidean metric $\delta_{i j}$. For example, $T^{a} T_{a}=T^{0} T_{0}-T^{i} T_{i}$. Covector components of torsion tensor have simple decompositions $\left(T_{a}\right):=\left(T_{0}, T_{i}\right)$ and $\left(T_{a}^{*}\right):=\left(T_{0}^{*}, T_{i}^{*}\right)$. In the massive case, components $T_{0}$ and $T_{0}^{*}$ describe scalar particles $0^{+}$and $0^{-}$, respectively, where indices denote parities. Covector components $\left(T_{i}\right)$ and $\left(T_{i}^{*}\right)$ correspond to vector particles of spin $1^{-}$and $1^{+}$. Irreducible component $S_{a b c}$ describes more particles. Because of the antisymmetry in the first two indices the following representations hold:

$$
S_{i j 0}=\varepsilon_{i j l} H^{l} \quad \Leftrightarrow \quad H^{l}:=\frac{1}{2} S_{i j 0} \varepsilon^{i j l} ; \quad S_{i j k}=\varepsilon_{i j l} H_{k}^{l} \quad \Leftrightarrow \quad H_{k}^{l}:=\frac{1}{2} S_{i j k} \varepsilon^{i j l},
$$

where $\varepsilon_{i j k}$ is the totally antisymmetric third rank tensor, $\varepsilon_{123}=1$. The relation $S_{i j k} \varepsilon^{i j k}=$ 0 implies the tracelessness condition $H^{i}{ }_{i}=0$. Components $S_{0 i j}$ are decomposed into symmetric traceless, antisymmetric parts and the trace:

$$
S_{0 i j}=S_{0\{i j\}}+S_{0[i j]}+\frac{1}{3} \delta_{i j} S, \quad S:=S_{0 i}{ }^{i} .
$$

Equality $S_{a 0}{ }^{a}=S_{00}{ }^{0}+S_{i 0}{ }^{i}=0$ implies $S=0$. From the relation $S_{a i}{ }^{a}=S_{0 i}{ }^{0}+S_{j i}{ }^{j}=0$ we deduce that

$$
S_{0 i 0}=-\varepsilon_{i j k} H^{j k} \quad \Leftrightarrow \quad H^{[m n]}=-\frac{1}{2} S_{0 i 0} \varepsilon^{i m n} .
$$

Finally, the equality $S_{0 i j}+S_{i j 0}+S_{j 0 i}=0$ yields the expression for the antisymmetric part

$$
S_{0[i j]}=-\frac{1}{2} \varepsilon_{i j k} H^{k} \quad \Leftrightarrow \quad H^{k}=-S_{0 i j} \varepsilon^{i j k}
$$

Thus, irreducible components of torsion with respect to the subgroup $\mathbb{S O}(3) \subset \mathbb{S O}(1,3)$ are:

$$
\begin{aligned}
& S_{0 i 0}, \quad\left(1^{-}\right)-3 \text { components, } \\
& S_{0\{i j\}}, \quad S_{0 i}{ }^{i}=0, \quad\left(2^{+}\right)-5 \text { components, } \\
& H_{\{i j\}}, \quad H_{i}{ }^{i}=0, \quad\left(2^{-}\right)-5 \text { components, } \\
& H_{i}, \quad\left(1^{+}\right)-3 \text { components, } \\
& T_{0}, \quad\left(0^{+}\right)-1 \text { component, } \\
& T_{i}, \quad\left(1^{-}\right)-3 \text { components, } \\
& T_{0}^{*}, \quad\left(0^{-}\right)-1 \text { component, } \\
& T_{i}^{*}, \quad\left(1^{+}\right)-3 \text { components, }
\end{aligned}
$$

the antisymmetric parts $S_{0[i j]}$ and $H_{[i j]}$ being expressed through independent components by equalities (11) and (10).

In the massive case, all independent $\mathbb{S O}(3)$-components of torsion are propagated. If one or more components of torsion turn out to be massless, then the model posses extra gauge symmetry and the number of propagated components is reduced but positive definiteness of the velocity quadratic form does not change. Therefore we focus our attention on the massive case. 


\section{Torsion mass terms}

Since $T_{a b c}$ has three Lorentz-irreducible components, $S_{a b c}, T_{a}$, and $T_{a}^{*}$, then the most general mass term depends on three constants:

$$
L_{m}:=-\beta_{1} S^{a b c} S_{a b c}-\beta_{2} T^{a} T_{a}-\beta_{3} T^{* a} T_{a}^{*} .
$$

If there are no tachyons, then this quadratic form must be negative definite. Simple calculations yield formulae:

$$
\begin{aligned}
S^{a b c} S_{a b c} & =2 S^{0\{i j\}} S_{0\{i j\}}-2 H^{\{i j\}} H_{\{i j\}}-3 S^{0 i 0} S_{0 i 0}+3 H^{i} H_{i}, \\
T^{a} T_{a} & =\left(T_{0}\right)^{2}-T^{i} T_{i}, \\
T^{* a} T_{a}^{*} & =\left(T_{0}^{*}\right)^{2}-T^{* i} T_{i}^{*} .
\end{aligned}
$$

We see that for any choice of $\beta_{1,2,3}$ the quadratic form (13) is not negative definite. It means that those torsion components which produce positive contribution must be nonpropagating and have no kinetic terms.

Suppose that $\beta_{1}>0$, then the negative contribution is given by $S_{0\{i j\}}$ and $H_{i}$. Thus, if the kinetic term is correct, the component $S_{a b c}$ can describe only massive particles $2^{+}$and $1^{+}$. Components $H_{\{i j\}}$ and $S_{0 i 0}$ produce positive contribution, correspond to tachyons, and must have no velocity squared terms in the Lagrangian. Conversely, if $\beta_{1}<0$, then propagating modes of torsion component $S_{a b c}$ can be only $H_{\{i j\}}$ and $S_{0 i 0}$. In this case, $S_{a b c}$ describes only particles $2^{-}$and $1^{-}$.

Vector $T_{a}$ and pseudovector $T_{a}^{*}$ components of torsion describe particles of positive mass in the following cases. If $\beta_{2}>0$, then propagating mode can be only $T_{0}$, which correspond to scalar particles $0^{+}$. For $\beta_{2}<0$ the negative contribution is given by vector component $T_{i}$ describing $1^{-}$particles.

The similar situation happens for $T_{a}^{*}$ component. If $\beta_{3}>0$ or $\beta_{3}<0$, then propagating particles can be only $T_{0}^{*}\left(0^{-}\right)$or $T_{i}^{*}\left(1^{+}\right)$, respectively.

\section{$5 \quad$ Kinetic terms}

The kinetic term has dimensionality $l^{-4}$ and is built from tensors [10]:

$$
\begin{array}{llll}
\nabla_{\alpha} \nabla_{\beta} R_{\gamma \delta \epsilon \zeta}, & R_{\alpha \beta \gamma \delta} R_{\epsilon \zeta \eta \theta}, & \nabla_{\alpha} \nabla_{\beta} \nabla_{\gamma} T_{\delta \epsilon \zeta}, & \nabla_{\alpha} T_{\beta \gamma \delta} \nabla_{\epsilon} T_{\zeta \eta \theta}, \\
\nabla_{\alpha} \nabla_{\beta} T_{\gamma \delta \epsilon} T_{\zeta \eta \theta}, & \nabla_{\alpha} T_{\beta \gamma \delta} T_{\epsilon \zeta \eta} T_{\theta \iota \kappa}, & T_{\alpha \beta \gamma} T_{\delta \epsilon \zeta} T_{\eta \theta \iota} T_{\kappa \lambda \mu}, & \nabla_{\alpha} T_{\beta \gamma \delta} R_{\epsilon \zeta \eta \theta}, \\
T_{\alpha \beta \gamma} \nabla_{\delta} R_{\epsilon \zeta \eta \theta}, & T_{\alpha \beta \gamma} T_{\delta \epsilon \zeta} R_{\eta \theta \iota \kappa}, & &
\end{array}
$$

There are 151 invariants listed in [10] which are too many for analytical analysis. Nevertheless, note the following. The velocities squared terms $\dot{T}^{2}$, where the dot denote time derivatives $\partial / \partial x^{0}$ and tensor indices are dropped, are contained in invariants $R^{2}, \nabla T \nabla T$, and $\nabla T R$, and also in invariants $\nabla \nabla T T$ and $\nabla T R$ after integration by parts. In the linear approximation, all invariants quadratic in velocities have the same form as invariants $\nabla T \nabla T$. Therefore, it is sufficient to consider only invariants constructed from the tensor $\nabla_{\alpha} T_{\beta \gamma \delta} \nabla_{\epsilon} T_{\zeta \eta \theta}$ by different contractions with the metric.

We start with the component $S_{a b c}$. Because this component satisfies Eqs. (8), there are only three independent invariants with respect to the full Lorentz group including 
reflections which are quadratic in first derivatives. We choose them in the form

$$
\nabla_{a} S_{b c d} \nabla^{a} S^{b c d}, \quad \nabla_{a} S_{b}{ }^{a}{ }_{c} \nabla_{d} S^{b d c} \quad \nabla_{a} S_{b}{ }^{a}{ }_{c} \nabla_{d} S^{c d b}
$$

The remaining invariants can be expressed through them. The proof of this statement is by item-by-item examination using the following properties. Two integration by parts imply relations:

$$
\begin{aligned}
& \nabla_{a} S_{b}{ }^{a}{ }_{c} \nabla_{d} S^{b d c} \stackrel{\stackrel{\text { div }}{=}}{=} \nabla_{a} S_{b c d} \nabla^{c} S^{b a d}, \\
& \nabla_{a} S_{b}{ }^{a}{ }_{c} \nabla_{d} S^{c d b} \stackrel{\text { div }}{=} \nabla_{a} S_{b c d} \nabla^{c} S^{d a b}, \\
& \nabla_{a} S_{b c}{ }^{a} \nabla_{d} S^{b c d} \stackrel{\text { div }}{=} \nabla_{a} S_{b c d} \nabla^{d} S^{b c a} .
\end{aligned}
$$

Moreover, Eq. (8) implies identities:

$$
\begin{aligned}
\nabla_{a} S_{b c d} \nabla^{a} S^{c d b} & =-\frac{1}{2} \nabla_{a} S_{b c d} \nabla^{a} S^{b c d} \\
\nabla_{[a} S_{b c] d} \nabla^{[a} S^{b c] d} & =\frac{1}{3} \nabla_{a} S_{b c d}\left(\nabla^{a} S^{b c d}+2 \nabla^{c} S^{a b d}\right) .
\end{aligned}
$$

Finally, there is the relation

$$
\begin{aligned}
0=\nabla_{a} S_{b c d}\left(\nabla^{b} S^{c d a}+\nabla^{c} S^{d b a}+\nabla^{d} S^{b c a}\right)= & \nabla_{a} S_{b c d}\left(\nabla^{d} S^{b c a}+2 \nabla^{c} S^{d b a}\right)= \\
& =\nabla_{a} S_{b c d}\left(\nabla^{d} S^{b c a}-2 \nabla^{c} S^{b a d}+2 \nabla^{c} S^{d a b}\right)
\end{aligned}
$$

where Eqs. (8) are used. The last equality after integration by parts is used to express the invariant $\nabla_{a} S_{b c}{ }^{a} \nabla_{d} S^{b c d}$ through invariants (16).

The absence of ghosts is defined by the velocity quadratic form. Therefore, we express invariants (16) through $(1+3)$-components and, for simplicity, keep only quadratic terms:

$$
\begin{array}{lll}
\nabla_{a} S_{b c d} \nabla^{a} S^{b c d} & \ni & 2 \dot{S}^{0\{i j\}} \dot{S}_{0\{i j\}}-2 \dot{H}^{\{i j\}} \dot{H}_{\{i j\}}-3 \dot{S}^{0 i 0} \dot{S}_{0 i 0}+3 \dot{H}^{i} \dot{H}_{i}, \\
\nabla_{a} S_{b}{ }^{a}{ }_{c} \nabla_{d} S^{b d c} & \ni & \dot{S}^{0\{i j\}} \dot{S}_{0\{i j\}}-\dot{S}^{0 i 0} \dot{S}_{0 i 0}+\frac{1}{2} \dot{H}^{i} \dot{H}_{i}, \\
\nabla_{a} S_{b}{ }^{a}{ }_{c} \nabla_{d} S^{c d b} & \ni & \dot{S}^{0\{i j\}} \dot{S}_{0\{i j\}}-\frac{1}{2} \dot{H}^{i} \dot{H}_{i} .
\end{array}
$$

The kinetic part of the Lagrangian for torsion component $S_{a b c}$ has the form

$$
L_{\mathrm{K}}:=c_{1} \nabla_{a} S_{b c d} \nabla^{a} S^{b c d}+c_{2} \nabla_{a} S_{b}{ }^{a}{ }_{c} \nabla_{d} S^{b d c}+c_{3} \nabla_{a} S_{b}{ }^{a}{ }_{c} \nabla_{d} S^{c d b}
$$

where $c_{1,2,3}$ are coupling constants. In terms of $(1+3)$-decomposition this Lagrangian yields the quadratic form

$$
\begin{aligned}
L_{\mathrm{K}}^{(2)}=\dot{S}^{0\{i j\}} \dot{S}_{0\{i j\}}\left(2 c_{1}+c_{2}+c_{3}\right) & -2 \dot{H}^{\{i j\}} \dot{H}_{\{i j\}} c_{1}- \\
& -\dot{S}^{0 i 0} \dot{S}_{0 i 0}\left(3 c_{1}+c_{2}\right)+\dot{H}^{i} \dot{H}_{i}\left(3 c_{1}+\frac{1}{2} c_{2}-\frac{1}{2} c_{3}\right) .
\end{aligned}
$$

The analysis of the mass term for $S_{a b c}$ shows that propagating can be either separate torsion $\mathbb{S O}(3)$-components or pairs $\left(S_{0\{i j\}}, H_{i}\right)$ or $\left(H_{\{i j\}}, S_{0 i 0}\right)$. 
Since we have only three invariants (16), and there are four independent $\mathbb{S O}(3)$ components of torsion $S_{a b c}$, then the absence of three kinetic terms implies the absence of the fourth. Therefore Lagrangian (18) cannot describe propagation of only one $\mathbb{S O}(3)$ irreducible component of $S_{a b c}$.

Now we consider the possibility of simultaneous propagation of two $\mathbb{S O}(3)$-irreducible components of $S_{a b c}$. If the only propagating components are $S_{0\{i j\}}$ and $H_{i}$, then the following equalities must hold

$$
c_{1}=0, \quad c_{2}=0,
$$

the constant $c_{3}$ being arbitrary. Then the kinetic part of the Lagrangian is

$$
L_{\mathrm{K}}^{(2)}=c_{3} \dot{S}^{0\{i j\}} \dot{S}_{0\{i j\}}-\frac{1}{2} c_{3} \dot{H}^{i} \dot{H}_{i}
$$

It is clear that for any $c_{3} \neq 0$ the model contains ghost. Consequently, propagation of only $S_{0\{i j\}}$ and $H_{i}$ is impossible.

Similar situation happens with components $H_{\{i j\}}, S_{0 i 0}$. If they are the only propagating ones, then the equalities

$$
c_{3}=2 c_{1}, \quad c_{2}=-4 c_{1}
$$

must hold, where $c_{1}$ is arbitrary. The corresponding kinetic part of the Lagrangian is

$$
L_{\mathrm{K}}^{(2)}=-2 c_{1} \dot{H}^{\{i j\}} \dot{H}_{\{i j\}}+c_{1} \dot{S}^{0 i 0} \dot{S}_{0 i 0}
$$

We see that the model contains ghost for any $c_{1} \neq 0$. Therefore Lagrangian $L_{\mathrm{K}}^{(2)}$ cannot describe propagation of only one pair of components $\left(H_{\{i j\}}, S_{0 i 0}\right)$.

Thus, there is no Lagrangian in the considered class which propagates the component $S_{a b c}$ without ghosts and tachyons.

Now we discuss the irreducible component $T_{a}$. It is the usual vector field. There are two possibilities of models without ghosts and tachyons. Lagrangians

$$
\begin{aligned}
L_{1} & :=-\frac{1}{4} \gamma_{1} F^{a b} F_{a b}+\frac{1}{2} m_{1}^{2} T^{a} T_{a}, \\
L_{2} & :=\frac{1}{2} \gamma_{2}\left(\nabla_{a} T^{a}\right)^{2}-\frac{1}{2} m_{2}^{2} T^{a} T_{a},
\end{aligned}
$$

where $F_{a b}:=\partial_{a} T_{b}-\partial_{b} T_{a}, \gamma_{1,2}>0$ and $m_{1,2}>0$ are the only ones without ghosts and tachyons. Lagrangian (20) describes propagation of vector particles $1^{-}$of mass $m_{1}$. It is the Proca field. The second Lagrangian (21) propagates scalar particles $0^{+}$of mass $m_{2}$.

The same happens for pseudovector torsion component $T_{a}^{*}$. There are only two ghost and tachyon free Lagrangians:

$$
\begin{aligned}
L_{3} & :=-\frac{1}{4} \gamma_{3} G^{a b} G_{a b}+\frac{1}{2} m_{3}^{2} T^{* a} T_{a}^{*}, \\
L_{4} & :=\frac{1}{2} \gamma_{4}\left(\nabla_{a} T^{* a}\right)^{2}-\frac{1}{2} m_{4}^{2} T^{* a} T_{a}^{*},
\end{aligned}
$$

where $G_{a b}:=\partial_{a} T_{b}^{*}-\partial_{b} T_{a}^{*}, \gamma_{3,4}>0$ and $m_{3,4}>0$. Lagrangians (22) and (23) do not contain ghosts and tachyons and propagate particles $1^{+}$and $0^{-}$of masses $m_{3}$ and $m_{4}$, respectively. 
Returning to the gravity models with dynamical models, we summarize. We have found four Lagrangians

$$
\begin{aligned}
& \frac{1}{\sqrt{|g|}} L_{13}:=\kappa \widetilde{R}(g)-\frac{1}{4} \gamma_{1} F^{\alpha \beta} F_{\alpha \beta}+\frac{1}{2} m_{1}^{2} T^{\alpha} T_{\alpha}-\frac{1}{4} \gamma_{3} G^{\alpha \beta} G_{\alpha \beta}+\frac{1}{2} m_{3}^{2} T^{* \alpha} T_{\alpha}^{*}, \\
& \frac{1}{\sqrt{|g|}} L_{23}:=\kappa \widetilde{R}(g)+\frac{1}{2} \gamma_{2}\left(\nabla_{\alpha} T^{\alpha}\right)^{2}-\frac{1}{2} m_{2}^{2} T^{\alpha} T_{\alpha}-\frac{1}{4} \gamma_{3} G^{\alpha \beta} G_{\alpha \beta}+\frac{1}{2} m_{3}^{2} T^{* \alpha} T_{\alpha}^{*}, \\
& \frac{1}{\sqrt{|g|}} L_{14}:=\kappa \widetilde{R}(g)-\frac{1}{4} \gamma_{1} F^{\alpha \beta} F_{\alpha \beta}+\frac{1}{2} m_{1}^{2} T^{\alpha} T_{\alpha}+\frac{1}{2} \gamma_{4}\left(\nabla_{\alpha} T^{* \alpha}\right)^{2}+\frac{1}{2} m_{4}^{2} T^{* \alpha} T_{\alpha}^{*} \\
& \frac{1}{\sqrt{|g|}} L_{24}:=\kappa \widetilde{R}(g)+\frac{1}{2} \gamma_{2}\left(\nabla_{\alpha} T^{\alpha}\right)^{2}-\frac{1}{2} m_{2}^{2} T^{\alpha} T_{\alpha}+\frac{1}{2} \gamma_{4}\left(\nabla_{\alpha} T^{* \alpha}\right)^{2}+\frac{1}{2} m_{4}^{2} T^{* \alpha} T_{\alpha}^{*} .
\end{aligned}
$$

For $\kappa>0, \gamma_{1,2,3,4}>0, m_{1,2,3,4}>0$ these Lagrangians do not contain ghosts and tachyons. In all four cases, the first term is the usual Hilbert-Einstein Lagrangian describing the massless graviton $2^{+}$. Lagrangians $L_{13}, L_{23}, L_{14}$, and $L_{24}$ propagate in addition massive degrees of freedom: $\left(1^{-}, 1^{+}\right),\left(0^{+}, 1^{+}\right),\left(1^{-}, 0^{-}\right)$, and $\left(0^{+}, 0^{-}\right)$, respectively.

Mass term in the obtained Lagrangians can be set to zero. Then torsion describes massless particles of the same spins and parities. The extra gauge symmetries appear in these cases, and the number of propagating degrees of freedom reduces. For example, let us put $m_{1}=0$ in $L_{13}$ and $L_{14}$. Then these Lagrangians are invariant with respect to gauge transformations

$$
T_{\alpha} \mapsto T_{\alpha}+\partial_{\alpha} \varphi
$$

where $\varphi(x)$ is an arbitrary local transformation parameter. These transformations coincide with the gauge transformations in electrodynamics. Therefore, if we switch on the interaction with matter fields in the same way, then the torsion trace $T_{a}$ can be identified with the electromagnetic potential. Unfortunately, the geometric meaning of such identification is not clear and requires further analysis.

If $m_{2}=0$ in Lagrangians $L_{23}$ and $L_{24}$, then they are invariant with respect to gauge transformations

$$
T^{\alpha} \mapsto T^{\alpha}+\widetilde{\nabla}_{\beta} \omega^{\beta \alpha},
$$

where $\omega^{\beta \alpha}(x)=-\omega^{\alpha \beta}(x)$ are local parameters. Note that the number of independent parameters is

$$
C_{4}^{2}-C_{4}^{3}+C_{4}^{4}=6-4+1=3,
$$

which is necessary for elimination of three unphysical degrees of freedom of vector field $T^{\alpha}$.

The same happens with the pseudovector field $T_{a}^{*}$.

\section{Conclusion}

Thus, supposing that kinetic terms of vierbein $e_{\alpha}{ }^{a}$ and torsion $T_{\alpha \beta \gamma}$ do not mix, we proved that there are only four Lagrangians $L_{13}, L_{23}, L_{14}$, and $L_{24}$ without ghosts and tachyons. In all cases, the vierbein describes one massless graviton $2^{+}$. In addition, torsion describes massive or massless particles $1^{-}, 1^{+}\left(L_{13}\right) ; 0^{+}, 1^{+}\left(L_{23}\right) ; 1^{-}, 0^{-}\left(L_{14}\right)$; or $0^{+}, 0^{-}\left(L_{24}\right)$. Moreover, we proved that any other Lagrangian for torsion free of ghosts and tachyons must have the same linear approximation as one of the listed. 
We proved also that the irreducible component $S_{a b c}$ of torsion cannot have a Lagrangian without ghosts and tachyons. The same result was obtained in [1] using different causal analysis. In addition, the causal analysis yields the argument in favor of Lagrangian (24) of the present paper [12.

This work was performed at the Steklov International Mathematical Center and supported by the Ministry of Science and Higher Education of the Russian Federation (agreement no. 075-15-2019-1614).

\section{References}

[1] F. W. Hehl, P. von der Heyde, G. D. Kerlick, and J. M. Nester. General relativity with spin and torsion: foundations and prospects. Rev. Mod. Phys., 48(3):393-416, 1976.

[2] F. W. Hehl, J. D. McCrea, E. W. Mielke, and Y. Ne'eman. Metric-affine gauge theory of gravity: Field equations, Noether identities, world spinors, and breaking of dilaton invariance. Phys. Rep., 258(1\&2):1-171, 1995.

[3] I. L. Shapiro. Physical aspects of the space-time torsion. Phys. Rep., 357:113-213, 2002. arXiv:1204.5505 [astro-ph.CO].

[4] M. Blagojević. Gravitation and Gauge Symmetries. IOP, Bristol and Philadelphia, 2002.

[5] S. Capozziello and M. De Laurentis. Extended theories of gravity. Phys. Rep., 509:167-321, 2011. arXiv:1204.5505 [astro-ph.CO].

[6] D. E. Neville. Gravity lagrangian with ghost-free curvature-squared terms. Phys. Rev., D18(10):3535-3543, 1978.

[7] E. Sezgin and P. van Nieuwenhuizen. New ghost-free gravity lagrangians with propagating torsion. Phys. Rev., D21(12):3269-3280, 1980.

[8] E. Sezgin. Class of ghost-free gravity lagrangians with propagating torsion. Phys. Rev., D24(6):1677-1680, 1981.

[9] K. Hayashi and T. Shirafuji. Gravity from Poincaré gauge theory of the fundamental particles. IV. Mass and energy of particle spectrum. Prog. Theor. Phys., 64(6):2222$2241,1980$.

[10] S. M. Christensen. Second- and fourth-order invariants on curved manifolds with torsion. J. Phys., A13(9):3001-3009, 1980.

[11] L. Fabbri and M. Tecchiolli. Restrictions on torsion-spinor field theory. Mod. Phys. Lett. A, 34:1950311, 2019. arXiv:1811.12060 [physics.gen-ph].

[12] L. Fabbri. A discussion on the most general torsion-gravity with electrodynamics for Dirac spinor matter fields. Int. J. Geom. Meth. Mod. Phys., 12:1550099, 2015. arXiv:1409.2007 [gr-qc]. 